\title{
Electronic origins of the giant volume collapse in the pyrite mineral MnS2
}

Durkee, Dylan; Smith, Dean; Torchio, Raffaella; Petitgirard, Sylvain; Briggs, Richard; Kantor, Innokenty; Evans, Shaun R.; Chatterji, Tapan; Irifune, Tetsuo; Pascarelli, Sakura

Total number of authors:

13

Published in:

Journal of Solid State Chemistry

Link to article, DOI:

10.1016/j.jssc.2018.10.032

Publication date:

2019

Document Version

Peer reviewed version

Link back to DTU Orbit

Citation (APA):

Durkee, D., Smith, D., Torchio, R., Petitgirard, S., Briggs, R., Kantor, I., Evans, S. R., Chatterji, T., Irifune, T., Pascarelli, S., Lawler, K. V., Salamat, A., \& Kimber, S. A. J. (2019). Electronic origins of the giant volume collapse in the pyrite mineral MnS. Journal of Solid State Chemistry, 269, 540-546.

https://doi.org/10.1016/j.jssc.2018.40.032

\section{General rights}

Copyright and moral rights for the publications made accessible in the public portal are retained by the authors and/or other copyright owners and it is a condition of accessing publications that users recognise and abide by the legal requirements associated with these rights.

- Users may download and print one copy of any publication from the public portal for the purpose of private study or research.

- You may not further distribute the material or use it for any profit-making activity or commercial gain

- You may freely distribute the URL identifying the publication in the public portal 


\title{
Electronic origins of the giant volume collapse in the pyrite mineral $\mathrm{MnS}_{2}$
}

\author{
Dylan Durkee, ${ }^{1}$ Dean Smith, ${ }^{1}$ Raffaella Torchio, ${ }^{2}$ Sylvain Petitgirard,${ }^{3}$ Richard Briggs, ${ }^{4,5}$ \\ Innokenty Kantor, ${ }^{6,2}$ Shaun R. Evans, ${ }^{7,2}$ Tapan Chatterji, ${ }^{8}$ Tetsuo Irifune, ${ }^{9}$ Sakura \\ Pascarelli, ${ }^{2}$ Keith V. Lawler ${ }^{10, *}$ Ashkan Salamat, ${ }^{11, \dagger}$ and Simon A. J. Kimber ${ }^{12,13, \ddagger}$ \\ ${ }^{1}$ Department of Physics and Astronomy and HiPSEC, \\ University of Nevada Las Vegas, Las Vegas, Nevada 89154, USA \\ ${ }^{2}$ European Synchrotron Radiation Facility (ESRF), \\ 6 rue Jules Horowitz, BP 220, 38043 Grenoble Cedex 9, France. \\ ${ }^{3}$ Bayerisches Geoinstitut, University of Bayreuth, 95440 Bayreuth, Germany. \\ ${ }^{4}$ Present address: Lawrence Livermore National Laboratory, \\ 7000 East Avenue, Livermore, California 94550, USA \\ ${ }^{5}$ SUPA, School of Physics and Astronomy 8 Centre for Science at Extreme Conditions, \\ The University of Edinburgh, Edinburgh, EH9 3FD, UK \\ ${ }^{6}$ Technical University of Denmark, Department of Physics, 2800 Kgs. Lyngby, Denmark. \\ ${ }^{7}$ Present address: Philip Morris International, Neuchâtel, Switzerland \\ ${ }^{8}$ Institute Laue Langevin (ILL), 71 avenue des Martyrs, BP 220, 38043 Grenoble Cedex 9, France. \\ ${ }^{9}$ Geodynamics Research Center, Ehime University, Matsuyama, Japan. \\ ${ }^{10}$ Department of Chemistry and Biochemistry and HiPSEC, \\ University of Nevada Las Vegas, Las Vegas, Nevada 89154, USA. \\ ${ }^{11}$ Department of Physics and Astronomy and HiPSEC, \\ University of Nevada Las Vegas, Las Vegas, Nevada 89154, USA. \\ ${ }^{12}$ Université Bourgogne-Franche Comté, Université de Bourgogne, \\ ICB-Laboratoire Interdisciplinaire Carnot de Bourgogne, Bâtiment Sciences Mirande, \\ 9 Avenue Alain Savary, B-P. 47870, 21078 Dijon Cedex, France. \\ ${ }^{13}$ Formerly: Neutron Sciences Division, Oak Ridge National Laboratory, USA.
}

(Dated: October 15, 2018)

\begin{abstract}
The pyrite mineral $\mathrm{MnS}_{2}$ was recently shown to undergo a giant pressure-induced volume collapse at $\sim 12 \mathrm{GPa}$, via a disordered intermediate phase. The high pressure arsenopyrite phase is stabilised by metal-metal bonding, a mechanism now shown to be ubiquitous for $\mathrm{Mn}^{2+}$ chalcogenides. Here we report a spectroscopic investigation of this transition up to pressures of $22 \mathrm{GPa}$. Using XANES we show that the transition does not involve a change in oxidation state, consistent with the arsenopyrite crystal structure proposed at high pressure. Notably, the XANES spectrum is almost identical in the pressure-induced disordered phase, and after crystallisation induced by laser-heating. The former is therefore a 'valence bond glass', and is likely disordered due to kinetic hindrance of the phase transition. We also detect electronic changes in the compressed pyrite phase, and this is confirmed by Raman scattering which shows that the disulphide vibrations in the pyrite phase saturate before the volume collapse. Together with detailed DFT calculations, these results indicate that electronic changes precede valence bond formation between the $\mathrm{Mn}^{2+}$ cations.
\end{abstract}

PACS numbers: $64.60 . \mathrm{Cn}, 61.05 . \mathrm{C}$

\section{Statement of Copyright}

This manuscript has been authored by UT-Batelle LLC under Contract No. DE-AC05-000R22725 with the U.S. Department of Energy. The United States Government retains and the publisher, by accepting the article for publication, acknowledges that the United States Government retains a non-exclusive, paid-up, irrevocable, worldwide license to publish or reproduce the published form of this manuscript, or allow others to do so, for United States Government purposes. The Department of Energy will provide public access to these results of federally sponsored research in accordance with the DOE Public Access Plan (http://energy.gov/downloads/doe-public-access-plan).

\section{INTRODUCTION}

The evolution of electronic structure under pressure is important to a wide range of fields, from geology to superconductivity. The classic manifestation of such changes under pressure is a non-linear change (collapse) in unit cell volume at a critical pressure. The origins of these effects range from single-ion spin or valence transitions ${ }^{1,2}$, electron delocalisation ${ }^{3,4}$, to inter site charge transfer ${ }^{5}$ and bond formation ${ }^{6}$. However, even when advanced scattering and theory methods are exploited ${ }^{7,8}$, it is still often challenging to determine the driving force for such collapses in a given family of materials ${ }^{4}$. This is due to the interaction between the crystallographic lattice, spin/orbital degrees of 
freedom, and many body electron effects (e.g. metallisation). This is especially true when phase transitions occur via disordered intermediates, which hide the thermodynamically stable crystal structure at the critical pressure. A well known example is found in the pyrite mineral $\mathrm{MnS}_{2}$, which was first investigated at high pressure ${ }^{9,10}$ in 1985 . At ambient pressure, an insulating high-spin $\mathrm{Mn}^{2+}$ state is found, which is unstable when the pressure exceeds ca. $11 \mathrm{GPa}$. A strong dependence on experimental details was discovered, with an essentially amorphous phase discovered with synchrotron radiation. Using in-situ laser heating, we later showed ${ }^{11}$ that the thermodynamically stable high pressure phase is actually the arsenopyrite structure, with a giant volume collapse of $22 \%$ at 11 GPa. The high pressure phase hosts a notable dimerisation, with strongly alternating $\mathrm{Mn}-\mathrm{Mn}$ distances in the edge-shared $\mathrm{MnS}_{6}$ octahedral chains. This lead us to speculate that the formation of metal-metal bonds ${ }^{12}$ stabilised the large reduction in unit cell volume, and that this went beyond the standard single ion paradigm for spin state transitions. More recently, several discoveries have been made that lend weight to our predictions. Firstly, the simple rocksalt materials MnS and MnSe were shown to similarly collapse around $30 \mathrm{GPa}$ to a locally bonded phase ${ }^{13}$. Likewise, several $\mathrm{Mn}^{2+} \mathrm{honeycomb}$ compounds were also shown to dimerise ${ }^{14}$, all with significant volume reductions and the formation of short Mn-Mn distances. The apparent ubiquity of this mechanism for $\mathrm{Mn}^{2+}$ compounds is exciting, yet important questions remain unanswered. For example, huge hysteresis was discovered in $\mathrm{MnS}_{2}$, and the thermodynamic boundaries between the pyrite and arsenopyrite phases are unknown. Furthermore, decomposition ${ }^{15}$ to $\mathrm{MnS}+\mathrm{S}$ is found at ambient pressure

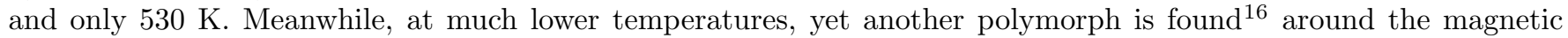
ordering temperature of $48 \mathrm{~K}$. The ordering temperature has been shown to sharply rise under pressure ${ }^{17}$, however its possible intersection with the arsenopyrite polymorph is unknown. The vibrational and electronic properties of the high pressure phase are also unknown, although DFT calculations were originally used to speculate that it would be insulating. Finally, and most mysteriously, it is unknown if the disordered intermediate formed on cold compression is the result of decomposition, as seen in e.g framework materials ${ }^{18}$, or simply because the transition is kinetically hindered due to the large volume change. To answer these questions, we have performed various spectroscopic investigations through the phase transition, in particular XANES and Raman, which are sensitive to the electronic structure and lattice vibrations respectively. While not directly probed by the measurements reported here, the question of metallisation at high pressure is briefly addressed. This is because the transport measurements of Wang et al detected metallic conduction in MnS, MnSe etc. This contradicts a simple picture of localised, bonded valence electrons, as well as the robust insulating gap found in DFT for the high pressure phase of $\mathrm{MnS}_{2}$.

\section{EXPERIMENTAL}

We used the same well-characterised natural sample of $\mathrm{MnS}_{2}$ as employed in our previous investigations ${ }^{9-11,16}$. This was finely ground and loaded into diamond anvil cells for Raman and x-ray investigations. A total of six (1-6) different diamond anvil cell loadings were carried out, all with 300 micron culet diamonds. A small chip of ruby was loaded in each, to determine the cell pressure using fluorescence measurements ${ }^{19}$.

For XANES measurements: (1) Helium was used as the pressure-transmitting medium (PTM) for cold compression up to $20 \mathrm{GPa}$. (2) Neon was used as the PTM and the sample laser heated using the online $\mathrm{Nd}$ :YAG $((\lambda=1064$ $\mathrm{nm})$. For loading (2), the sample was laser heated with a Nd:YAG system at ESRF. XANES measurements were performed on the ID24 beam line of the European Synchrotron Radiation Facility (ESRF) in Grenoble, France ${ }^{20,21}$. Measurements were performed in transmission using a pink beam. In order to avoid spurions caused by the Bragg edges of the diamonds/sample, we used nano crystalline diamond anvils ${ }^{22}$. We calculated the XANES spectra for the high and low pressure phases of $\mathrm{MnS}_{2}$ using multiple scattering calculations, as implemented in FEFF ${ }^{23}$. Finally, post-laser heating, we collected diffraction data using the ID09A high pressure beam line ${ }^{54}$, also at the ESRF, with a wavelength of $0.41 \AA$. Diffraction data were analysed using the GSAS package ${ }^{24,25}$.

For Raman spectroscopy: (3) Helium was used as the PTM and the sample compressed at RT to $15 \mathrm{GPa}(\lambda=488$ $\mathrm{nm})$. (4) 4:1 methyl/ethyl alcohol was used as the PTM and the sample was compressed at RT, attempting to track the pyrite phase up to a final pressure of $18.5 \mathrm{GPa}(\lambda=514.5 \mathrm{~nm})$. (5) The sample was elevated on a thin layer of $\mathrm{NaCl}$ and gas loaded with neon as the PTM. Heating using a $\mathrm{CO}_{2}$ laser $(\lambda=10.6 \mu \mathrm{m})$ was carried out at $11 \mathrm{GPa}$, whilst collecting Raman Spectra in situ $(\lambda=488 \mathrm{~nm})$. (6) $\mathrm{NaCl}$ was used as the PTM and sample was laser heated $(\lambda=10.6 \mu \mathrm{m})$ at $14 \mathrm{GPa}$. Laser line for spectroscopy $((\lambda=514.5 \mathrm{~nm})$. For loadings $(5)$ and $(6)$ the samples were heated using a single sided $\mathrm{CO}_{2}$ laser at UNLV. Raman measurements were performed either on a Jobin-Yvon Horiba T64000 spectrometer system or a home built Raman system using Optigrate notch filters with a f/9 spectrometer. All measurements were carried out using laser powers of 2-5 $\mathrm{mW}$.

Plane-wave density functional theory (PW-DFT) ${ }^{26,27}$ simulations were done with the Vienna ab initio simulation package (VASP) version 5.4.1 using the $\mathrm{PBEsol}^{28}$ functional. The rotationally invariant, single parameter Hubbard $\mathrm{DFT}+\mathrm{U}^{29}$ correction $\left(\mathrm{U}_{\text {eff }}=3.9 \mathrm{eV}\right)$ was applied to the Mn $3 d$ states to account for the strongly correlated nature of those electrons. $3.9 \mathrm{eV}$ was selected for its demonstrated performance in predicting Mn oxides, and because that 

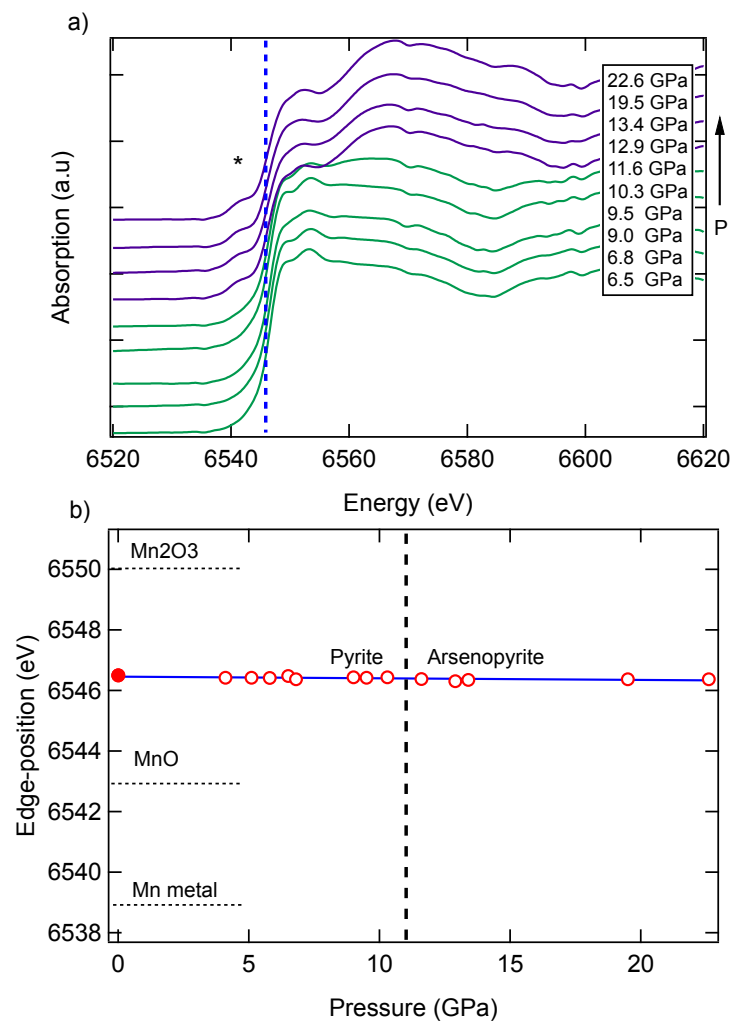

FIG. 1: (color online) (a) Pressure dependence of the XANES signal from $\mathrm{MnS}_{2}$ on cold compression. The emergence of a characteristic pre-peak feature is highlighted by an asterisk $\left(^{*}\right)$ and the edge position is marked by a dashed blue line; b) Pressure dependence of the Mn edge position, as parameterised by the derivative $d I / d E$. A linear fit shows that this does not vary over the entire pressure range studied. The dashed lines shows the ambient pressure edge range found for various Mn oxidation states.

combination of PBEsol+U and ferromagnetic (FM) ordering predicted volumes within $1 \%$ of the experimental values for the ambient pyrite and $20 \mathrm{GPa}$ arsenopyrite phases ${ }^{11,30}$. The vibrational calculations employed antiferromagnetic (AFM) type 1 ordering as it is the only antiferromagnetic ordering for on-atom localization that can be described with a single unit cell. While other orderings may have lower energy, this solution's geometry and electronic structure will be similar enough to make general conclusions. The Brillouin zone was represented by an automatically generated $\Gamma$-centered k-point grid with $0.025 \times 2 \pi \AA^{-1}$ resolution $^{32}$. Gaussian smearing was employed with a width of $0.05 \mathrm{eV}$. The basis set cutoff energy was $600 \mathrm{eV}$ using projector augmented wave (PAW) ${ }^{33}$ pseudo-potentials formulated for PBE GW with valence configurations: $3 s^{2} 3 p^{6} 4 s^{2} 3 d^{5}$ for $\mathrm{Mn}$ and $3 s^{2} 3 p^{4}$ for S. All structures were optimized at their respective pressure with energy tolerances of $1 \mathrm{E}-6 \mathrm{eV}$ and force tolerances $1 \mathrm{E}-2 \mathrm{eV} / \AA$. Vibrational calculations were conducted with density functional perturbation theory and processed with the phonopy package ${ }^{34}$.

\section{RESULTS AND DISCUSSIONS}

\section{X-ray absorption measurements}

We first describe the results of XANES experiments on the Mn $K$-edge. Compression was performed at room temperature, mimicking the pathway originally followed in Ref. 11 which produces a disordered phase, and recorded absorption edge data is shown in Fig. 1a. Changes in the oxidation state of Mn were investigated by tracking the edge position, which we parameterized by a Gaussian fit to the maximum in $d I / d E$. Fig. $1 \mathrm{~b}$ depicts the sub-eV change in absorption edge energy with pressure, alongside the expected (4-7 eV) change that would accompany a change in oxidation state. In this way, we rule out any gross pressure-induced redox reaction of the form $\mathrm{Mn}^{2+}+{S_{2}^{2-}}^{2} \rightarrow$ $\mathrm{Mn}^{4+}+2 \mathrm{~S}^{2-}$. In Fig. 1a, we also observe the onset of a pre-edge peak feature (asterisk) at higher pressure. Features 
a)
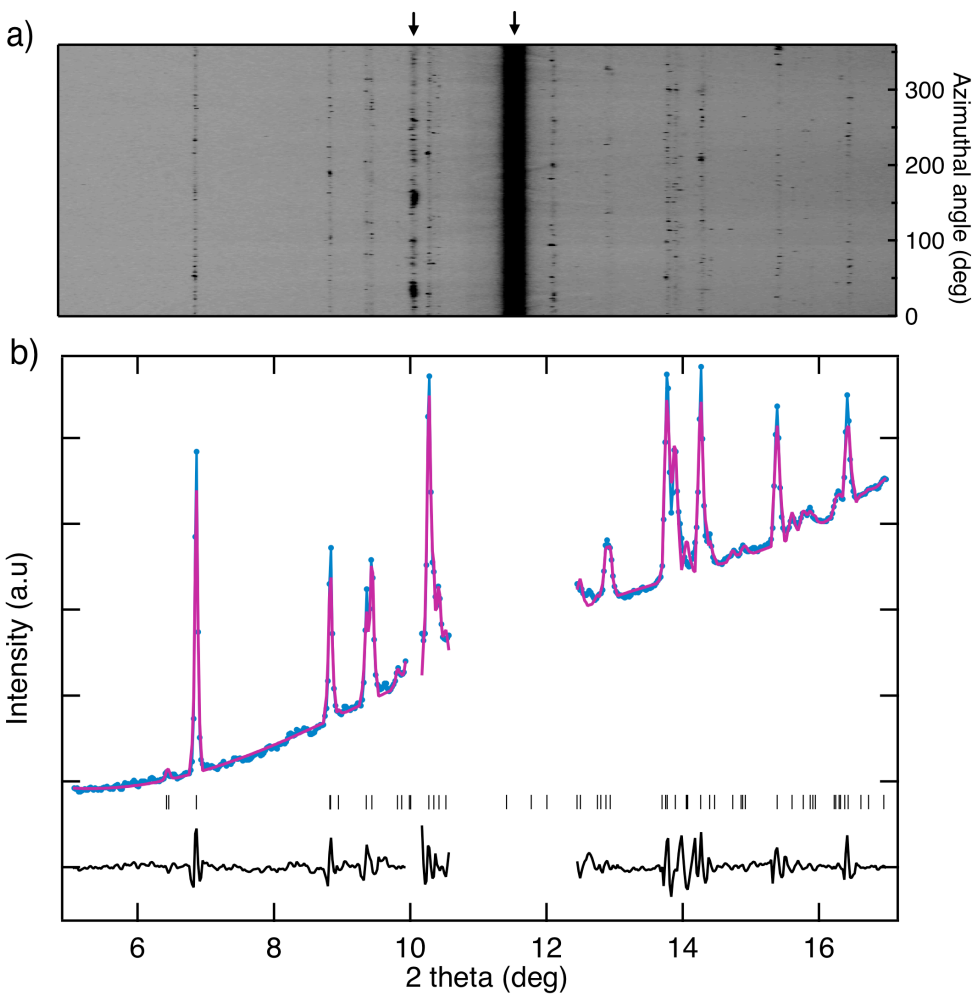

FIG. 2: (color online) a, top) Observed x-ray diffraction intensity for the laser heated sample of $\mathrm{MnS}_{2}$. The data are shown as a function of 2-theta and the azimuthal angle, which clearly highlights the strong Bragg line from the nano crystalline anvils. One other impurity line is also seen, just over $10^{\circ}$; b, bottom) When both of these features were excluded, a Le Bail intensity extraction using the $P 2_{1} / c$ arsenopyrite structure gave an excellent fit. This confirms that the bulk of the laser heated sample was the crystalline form of this polymorph. Note that the intensity of the peak from the nano crystalline anvils dwarfs the sample peaks by several orders of magnitude. The rather broad excluded region was therefore necessary to cut out the Bragg peak tails,which dominate over this 2-theta range.

such as this are indicative of transition metal coordination, oxidation and spin states, as discussed in more detail below, and the emergence of this pre-edge feature can be attributed to the onset of the transition beyond $P a \overline{3} \mathrm{MnS}_{2}$. We then crystallized the sample into its arsenopyrite $\left(P 2_{1} / c\right.$ structure at $22 \mathrm{GPa}$ by laser heating, as confirmed by synchrotron x-ray diffraction (Fig. 2). The large rising background in Fig. $2 \mathrm{~b}$ originates from the nanocrystalline diamond anvils employed in this experiment. As these anvils are strongly absorbing in the mid-infrared, the use of a $\mathrm{CO}_{2}$ laser to thermally anneal samples (as in Ref. 11 and other experiments presented here) was inhibited, and we employ instead a $1064 \mathrm{~nm} \mathrm{Nd:YAG} \mathrm{laser.} \mathrm{This} \mathrm{is} \mathrm{the} \mathrm{first} \mathrm{observation} \mathrm{of} \mathrm{laser-annealed} \mathrm{arsenopyrite} \mathrm{MnS}_{2}$ using this laser energy, and provokes an interesting discussion: typically materials which will absorb near-infrared radiation are metallic or semiconducting, and have plasma frequencies which result in high reflectivity in the mid-infrared - i.e. will not absorb $\mathrm{CO}_{2}$ laser radiation. The direct absorption of the $\mathrm{CO}_{2}$ laser light $\left(10.6 \mu \mathrm{m}, 943.4 \mathrm{~cm}^{-1}\right)$ in $\mathrm{MnS}_{2}$ can be attributed to the similar stretching mode frequencies of the $\mathrm{S}_{2}^{2-}$ dimers, providing activity in phonons from which the incident phonons may scatter to provide heat. This characteristic would persist into a putative metallic state, enabling similar absorption properties, and this observation does not contribute to the discussion on the transport properties of dimerised manganese chalcogenides under pressure.

To gain further insight into the signatures present in the XANES data, we now compare the data for the pyrite, disordered and crystallised arsenopyrite phases to our calculations. Within the limitations of the energy-dispersive XANES technique at pressure, as well as the simple approximations made, these provide a good guide to the pressure induced changes. For the pyrite phase (Fig. 3a), the agreement is fairly poor. However, the edge position, absence of a strong pre-peak, and gross features at higher energies are well reproduced. In contrast, the calculations works better for the arsenopyrite phase. The pre-peak is correctly predicted (although its intensity is overestimated), and the higher energy features are in phase with the data. The comparison of the calculated and observed (post laser heating) XANES spectra is shown in Fig. 3b. Good agreement in the edge position, pre-peak features, and higher 
a) b)
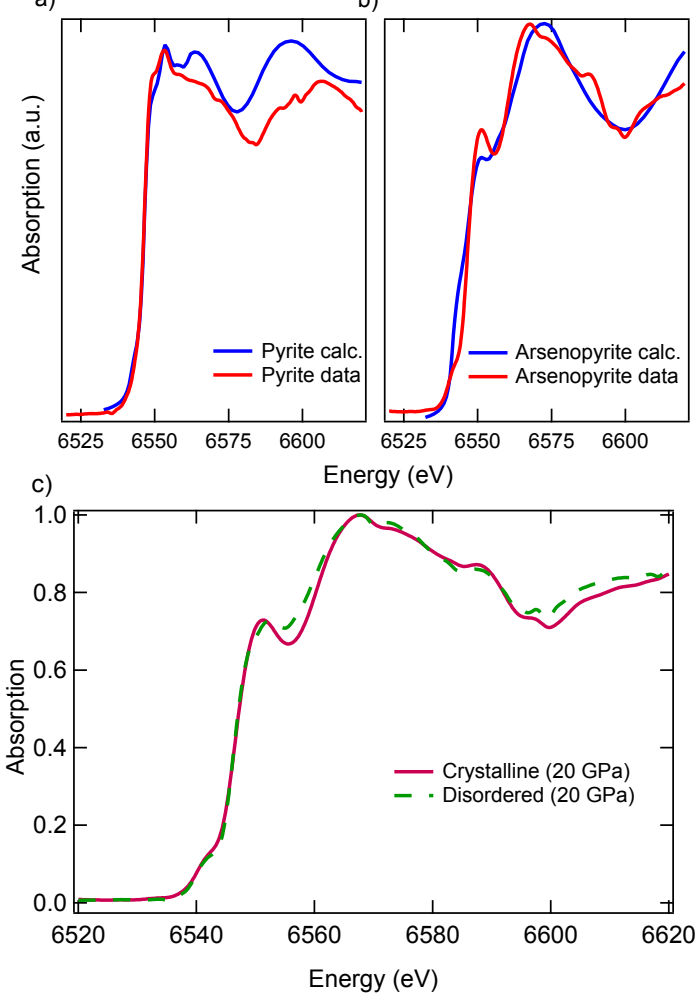

FIG. 3: (color online) (a) Comparison of the ambient pressure XANES data for pyrite( $\mathrm{Pa}-3$ ) MnS 2 with the results of DFT calculations; b) comparison of the laser-heated arsenopyrite $\left(\mathrm{P} 2{ }_{1} / \mathrm{c}\right) 20 \mathrm{GPa}$ XANES results to DFT calculations for the arsenopyrite phase; c) comparison of the XANES data for the pressure induced disordered and crystallised arsenopyrite phases at $20 \mathrm{GPa}$.

energy oscillation is observed. Even more striking is the comparison (Fig. 3c) between the crystalline and disordered phase at $20 \mathrm{GPa}$. These agree extremely well, which shows that the pressure-induced disordered phase of $\mathrm{MnS}_{2}$ is just a disordered form of the arsenopyrite phase. This strongly supports the kinetic hindrance hypothesis. Before moving on, we observe that the overall shape of the XANES spectra is remarkably similar ${ }^{35,36}$ to the Fe K-edge spectra of low-spin, semiconducting $\mathrm{FeS}_{2}$. In particular, we observe a broad oscillation after the edge, which was attributed to a resonance of Fe $p$-states in the continuum.

Having established the sensitivity of our XANES measurements to electronic changes in MnS $\mathrm{M}_{2}$ under pressure, we next looked at the pressurised pyrite region in more detail. These results are shown in Fig. 4. Our first observation is the strong pressure dependance of the post-edge region (6550-6560 eV, Fig. 4a) in the region of the transition. This begins to flatten strongly above $9 \mathrm{GPa}$, until the edge is almost a flat step just before the volume collapse (11.6 GPa). This is interesting, as standard DFT structure relaxation of $\mathrm{MnS}_{2}$ at pressure yields a metallic pyrite phase ${ }^{11}$, not the arsenopyrite structure. Earlier DFT work also shows that, in the absence of electron correlations, a low spin, metallic and reduced volume pyrite is the ground state ${ }^{37-39}$. Metallisation is also exactly what is found ${ }^{40,41}$ experimentally in pyrite $\mathrm{MnTe}_{2}$ above $8 \mathrm{GPa}$.

Also shown in Fig. 4b is the results of extracting the pre-peak features in $\mathrm{MnS}_{2}$. We fitted a pseudo-Voigt function to the edge jump, and subtracted this to yield this raw data. Surprisingly, this analysis also shows a weak pre-peak feature in the pyrite phase. In pure octahedral coordination (as found at ambient pressure), this is predicted to be extremely weak, as only a $1 s-3 d$ electric quadrupole transition is allowed ${ }^{42}$. Nevertheless, this becomes fairly obvious above $\sim 4 \mathrm{GPa}$ and its position can be tracked. We note that the pyrite crystal field is only perfectly $O_{h}$ for the nearest neighbour co-ordination sphere, and that next-nearest neighbour interactions may mix in a dipolar component. Additionally, any small distortion induced by pressure will also lead to an increase through this channel. The pre-peak in the pyrite phase is actually split into two components separated by $\sim 2 \mathrm{eV}$. In principle, this reflects the crystal field splitting $\left(10 \Delta_{0}\right)$ between the $t_{2 g}$ and $e_{g} d$-orbitals ${ }^{42,43}$. Indeed for FeS 2 , calculations ${ }^{46}$ give a ball-park figure of $3.5 \mathrm{eV}$. A possible lower bound for $\mathrm{MnS}_{2}$ is given by the results of electron energy loss spectroscopy ${ }^{47}$, which yield a splitting of $10 \Delta_{0}=0.9 \mathrm{eV}$. As shown in Fig. 4c, the doublet shifts in energy under pressure, ultimately 

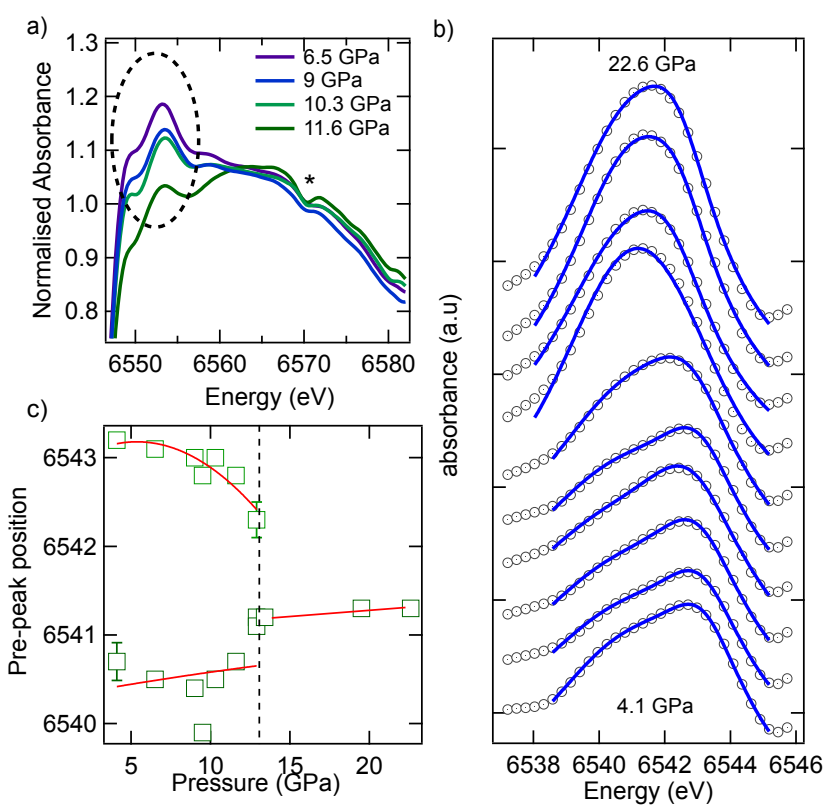

FIG. 4: (color online) (a) Pressure dependance of the edge region in the pyrite phase of $\mathrm{MnS}_{2}$. Note how the intensity is continually suppressed upon approaching the phase transition. The star marks a glitch from a Bragg feature from the cell or sample; b) Pressure dependence of the pre-edge feature extracted as described in the text. The pressure scale runs from 4 GPa for the bottom spectra to $22 \mathrm{GPa}$ for the top spectra, and lines are Lorentzian fits used to extract the positions. c) Obtained pressure dependence of the pre-edge position(s). Lines are guides to the eye.

coalescing into a single broad feature. This shift, which occurs at pressures well below the structural phase transition is a further indication of precursor electronic effects in the pyrite phase. As soon as the pressure induced disordered arsenopyrite is reached, the feature grows significantly in intensity and its position in energy becomes rather more stable as a function of pressure. It is important to note that the higher pressure data can be fitted with a single broad feature, or (with much larger uncertainties), a doublet. The $\mathrm{Mn}^{2+}$ oxidation state should always show a splitting of the pre-peak in $O_{h}$ symmetry, however, as is well known for oxides, this splitting becomes washed out as lower site symmetry mixes in a dipolar contribution from increased $3 d-p$ hybridisation ${ }^{43}$.

The position of pre-peaks in XANES analysis has widely been used as a proxy for spin-state transitions in iso-electronic $\mathrm{Fe}^{3+}$ materials. Recent examples include $\mathrm{Fe}_{2} \mathrm{O}_{3}$, also performed on the ID24 beam line by some of the present authors ${ }^{44}$. Here, the position of the Fe $K$-edge pre-peak was shown to track the collapse in Fe-O bond lengths extracted from EXAFS analysis. Although only the average pre-peak position was extracted throughout the entire pressure range, a doublet structure can also clearly been seen in the low-pressure phase, with a splitting of $\sim 2.5 \mathrm{eV}$, which is remarkably close to the crystal-field splitting of $2.75 \mathrm{eV}$ extracted for $\mathrm{Fe}^{3+}$ by optical means ${ }^{45}$. If we assume that the splitting we observe in $\mathrm{MnS}_{2}$ reflects the crystal field, then this immediately rules out simple toy models for the spin-state transition. Increasing pressure reduces ${ }^{11}$ the $<\mathrm{Mn}-\mathrm{S}>$ bond distance, which for an isolated octahedra would increase $10 \Delta_{0}$, favouring the low-spin state. However, in our case, the splitting reduces as a function of pressure. If the pyrite phase is heading towards a metallic ground state as hinted at by the XANES results and DFT calculations ${ }^{11}$, then many body effects may be more important in inducing the phase transition. This would be in contrast to the classic case of $\mathrm{MnO}$, where calculation comprehensively shows that a magnetic moment collapse induced by increasing crystal field drives the metal-insulator transition ${ }^{4}$.

\section{Vibrational spectroscopy}

The irreducible representations for the pyrite and arsenopyrite structures are well known, and have been reported by a number of authors ${ }^{48}$. For the purposes of the following section, we summarise them below. For pyrite, the crystal structure is cubic, $P a \overline{3}$, with $\Gamma=A_{g}+E_{g}+3 T_{g}+2 A_{u}+2 E_{u}+6 T_{u}$. This yields a total of five Raman active and eight IR active vibrations. These were assigned by Vogt et al, who used polarised Raman scattering on an orientated single crystal. These authors noted several features which indicate that $\mathrm{MnS}_{2}$ is fairly close to the ionic limit. This included the $\sim 240 \mathrm{~cm}^{-1}$ separation between the $A_{g}$ stretch and the $E_{g}$ libration, as well the presence of 
(a)
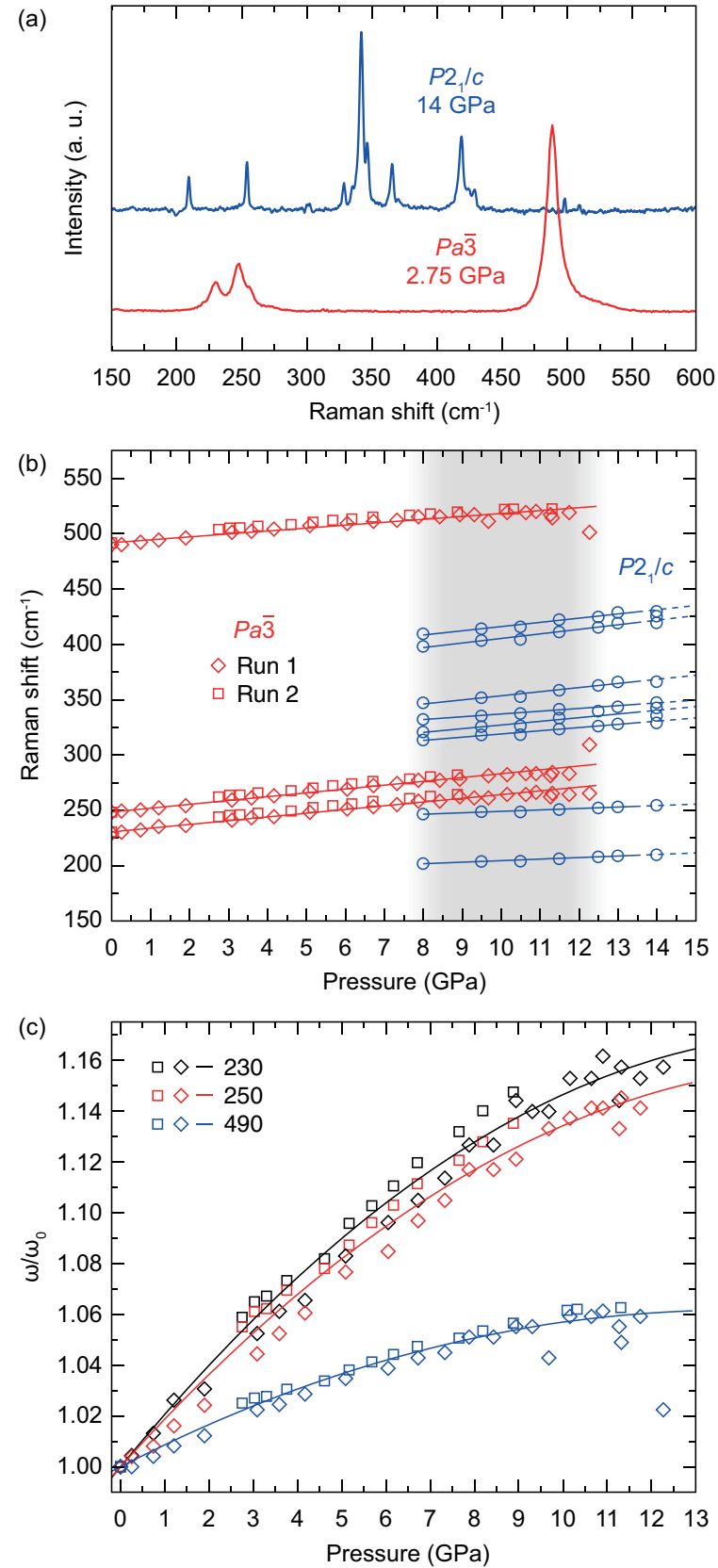

FIG. 5: (color online) a) Raman spectra recorded in the pyrite and arsenopyrite phases of $\mathrm{MnS}_{2}$. Note that the latter is post laser heating as described in the text; b) Pressure evolution of the main Raman frequencies of the pyrite (red symbols) and arsenopyrite (blue symbols) on decompression. The pyrite phase is measured upon compression using helium and methyl/ethyl pressure transmitting medium. The arsenopyrite phase is accessed at $14.5 \mathrm{GPa}$ following laser heating, and is followed here upon decompression. A broad region of phase coexistence is observed.; c) Pressure dependence of the main pyrite vibrations normalised to their ambient pressure values. Note that the features around $250 \mathrm{~cm}^{-1}$ are the $\mathrm{E}_{g}$ vibration, and the overtones from the intense $\mathrm{A}_{g}+\mathrm{T}_{g}$ feature at ca. $480 \mathrm{~cm}^{-1}$.

overtones and lack of significant $\left(\sim 1 \mathrm{~cm}^{-1}\right)$ Davydof splitting ${ }^{49}$ between the in phase $\mathrm{A}_{g}$ stretch and the $\mathrm{T}_{g}$ out of phase stretch. These features were said to make $\mathrm{MnS}_{2}$ the closest of the transition metal pyrites to an ionic molecular crystal. As shown in Fig. 5a, our powder averaged Raman spectra, which were measured on the same sample, are in good agreement with these results. As discussed below, the energy width of the excitations is significantly larger than the resolution function of the spectrometer. This is likely caused by strain effects on decompression, as previously reported for the recovered pyrite phase ${ }^{11}$ 
TABLE I: A comparison of the simulated and experimental vibrational modes for $P 2_{1} / c \mathrm{MnS}_{2}$ at $16 \mathrm{GPa}$.

\begin{tabular}{|c|c|c|c|c|c|c|c|c|c|}
\hline \multicolumn{6}{|c|}{ Raman Active Modes } & \multicolumn{4}{|c|}{ Infrared Active Modes } \\
\hline Symmetry & Exp. & Calculations & Symmetry & Exp. & Calculations & Symmetry & Calculations & Symmetry & Calculations \\
\hline $\mathrm{A}_{g}^{1}$ & 209.7 & 182.8 & $\mathrm{~B}_{g}^{1}$ & 209.7 & 182.8 & $\mathrm{~A}_{u}^{1}$ & 209.4 & $\mathrm{~B}_{u}^{1}$ & 330.8 \\
\hline $\mathrm{A}_{g}^{2}$ & 254.2 & 233.1 & $\mathrm{~B}_{g}^{2}$ & 254.2 & 233.2 & $\mathrm{~A}_{u}^{2}$ & 272.4 & $\mathrm{~B}_{u}^{2}$ & 374.1 \\
\hline $\mathrm{A}_{g}^{3}$ & 329.0 & 314.7 & $\mathrm{~B}_{g}^{3}$ & 329.0 & 314.7 & $\mathrm{~A}_{u}^{3}$ & 371.8 & $\mathrm{~B}_{u}^{3}$ & 376.4 \\
\hline $\mathrm{A}_{g}^{4}$ & 335.6 & 318.4 & $\mathrm{~B}_{g}^{4}$ & 342.4 & 323.6 & $\mathrm{~A}_{u}^{4}$ & 376.4 & $\mathrm{~B}_{u}^{4}$ & 401.6 \\
\hline $\mathrm{A}_{g}^{5}$ & 347.3 & 352.3 & $\mathrm{~B}_{g}^{5}$ & 366.1 & 360.8 & $\mathrm{~A}_{u}^{5}$ & 381.2 & $\mathrm{~B}_{u}^{5}$ & 404.2 \\
\hline $\mathrm{A}_{g}^{6}$ & 366.1 & 360.8 & $\mathrm{~B}_{g}^{6}$ & 419.4 & 414.1 & $\mathrm{~A}_{u}^{6}$ & 404.2 & $\mathrm{~B}_{u}^{6}$ & 445.8 \\
\hline $\mathrm{A}_{g}^{7}$ & 419.4 & 414.1 & $\mathrm{~B}_{g}^{7}$ & 425.4 & 435.0 & $\mathrm{~A}_{u}^{7}$ & 445.8 & $\mathrm{~B}_{u}^{7}$ & 478.9 \\
\hline $\mathrm{A}_{g}^{8}$ & 429.6 & 437.1 & $\mathrm{~B}_{g}^{8}$ & $\mathrm{X}$ & 474.4 & $\mathrm{~A}_{u}^{8}$ & 485.4 & & \\
\hline $\mathrm{A}_{g}^{9}$ & $\mathrm{X}$ & 474.4 & $\mathrm{~B}_{g}^{9}$ & $\mathrm{X}$ & 484.6 & & & & \\
\hline
\end{tabular}

Fig. 5a also contains the results of Raman spectroscopy on a crystallised sample of $\mathrm{MnS}_{2}$ in the arsenopyrite phase. This was prepared in a similar manner to our earlier work, and the data shown is at 16 GPa. This spectra is sharper than that of the decompressed pyrite phase, as expected from the highly crystalline laser-heated arsenopyrite (Fig. $2)$. For the arsenopyrite structure, the monoclinic $P 2_{1} / c$ structure yields ${ }^{50} \Gamma=9 A_{g}+9 B_{g}+9 A_{u}+9 B_{u}$. Sixteen of the 18 Raman active modes in isostructural $\mathrm{CoSb}_{2}$ were assigned by Larazević et al relying upon DFT vibrational calculations $^{50}$. Similar DFT simulations on $P 2_{1} / c \mathrm{MnS}_{2}$ produced a similar set of modes that are in reasonably good agreement with our experimental spectra, Table I. The assignment of the modes is very similar to the work on $\mathrm{CoSb}_{2}$, with the notable exception of a shift higher in energy owing to the lighter masses of the elements involved.

We now turn to our pressure dependent results, which were obtained on decompressing the laser heated arsenopyrite phase and yielded the results shown in Fig. 5b. We note that we also collected data on cold-compression of the pyrite phase, which were entirely consistent with these results, excepting the wipe-out of the Raman signal upon entering the disordered phase (not shown). These results highlight the different pressure dependance of the intense $A_{g}$ stretching feature at around $480 \mathrm{~cm}^{-1}$ and the $E_{g}$ disulphide libration around $250 \mathrm{~cm}^{-1}$. Although x-ray diffraction shows that the disulphide bond length barely changes with pressure ${ }^{11}$, the frequency of the former is seen to plateau around $10 \mathrm{GPa}$ (Fig. 5c). This implies that either the bond strength is changing, or that the vibration becomes increasingly hindered at pressure. This is in marked contrast to the nearly linear dependence of this feature in $\mathrm{FeS}_{2}$ when compressed ${ }^{51}$ to pressures of $50 \mathrm{GPa}$. We note that charge density measurements ${ }^{52,53}$ on $\mathrm{MnS}_{2}$ show that the $3 d \mathrm{Mn}^{2+}$ orbitals are mixed with electron density attributed to the $3 p \pi^{*}$ antibonding orbitals of the disulphide units. Our band structure and partial density of states calculations confirm this mixing. Pressure strongly reduces the Mn-S distance, increasing the overlap between these. This eventually raises the crystal field splitting sufficiently to favour a low-spin state. However, we also speculate that pressure increases back-bonding, weakening the disulphide bond. This was highlighted with the formation of the sulphur, which has a very large Raman scattering cross-section and therefore easily observed when present, as a decomposition product when any attempt was made to access the arsenopyrite phase at any pressure below 11.6 GPa. This pressure threshold confirms the crossover into the thermodynamic ground state of the arsenopyrite phase and the pressure sensitivity of thermal annealing with respect to the weakening the disulphide bond.

\section{DFT calculations}

Our DFT calculations of the pyrite phase are consistent with earlier results at ambient pressure. AFM type 3 ordering is preferred, but the other magnetic orderings (FM, AFM type 1, and AFM type 2) are very close energetically. AFM type 1, the second most favorable, is only $3.2 \mathrm{meV}$ per formula unit higher in energy. All the magnetic orderings exhibit an insulating gap (Fig. 6), with the tails of the half-occupied and half-unoccupied atomic-like Mn $3 d$ states separated by around $0.6 \mathrm{eV}$. Increasing the pressure on the system has the effect of lowering the relative energy of the unoccupied $3 d$ states, providing a gap of $\sim 0.4 \mathrm{eV}$ by $11 \mathrm{GPa}$ (Fig. $6 \mathrm{~b}$ ) for AFM type 2 and 3 ordering. In FM and AFM type 1 ordering, the gap between the tails of the occupied and unoccupied densities closes by 11 GPa. While the $\mathrm{Mn}$ is still $\mathrm{S}=5 / 2$, a small reduction in the magnetic moment is seen, indicating the onset of a change in the $d$ states. Increasing the pressure further to $20 \mathrm{GPa}$ causes the AFM type 3 structure to go metallic (Fig. 6c). In every other magnetic ordering each $\mathrm{Mn}$ transformed into the low-spin, $\mathrm{S}=1 / 2$ state (similar to Fig. 6d). The other magnetic orderings are more energetically favorable than AFM type 3, and the gap between the occupied and unoccupied $d$ 


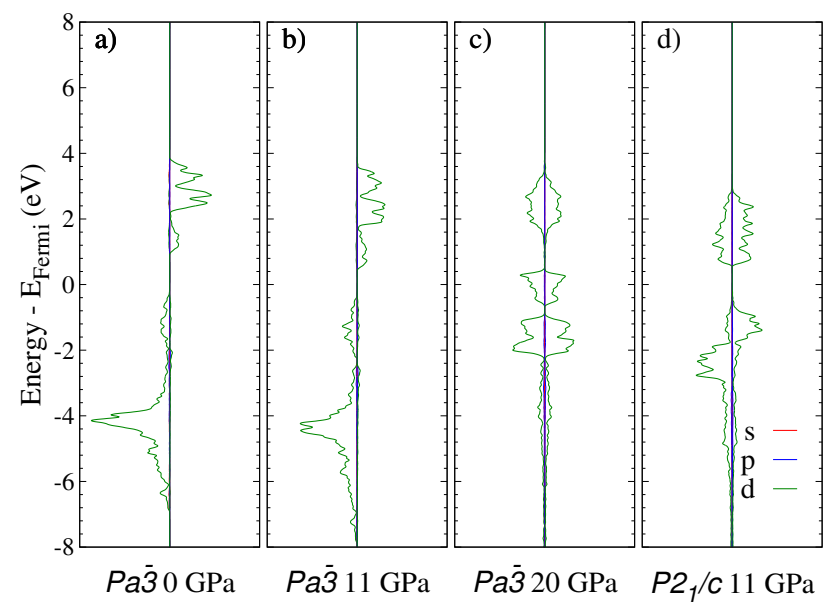

FIG. 6: (color online) A comparison of the computed site and angular momentum projected partial densities of states for a spin-up Mn atom (AFM type 3) in: a) 0 GPa pyrite, b) 11 GPa pyrite, c) 20 GPa pyrite, and d) 11 GPa arsenopyrite. Spin-up states are to the left, spin-down to the right. A spin-down Mn has an identical partial density of states, but with the spin-up and spin-down components reversed.

states increases $(\sim 0.4 \mathrm{eV})$. From those results it can be inferred that the metallisation $P a \overline{3} \mathrm{MnS}_{2}$ requires at least a 2 step electronic transition pathway; the low-spin state for AFM type 3 pyrite $\mathrm{MnS}_{2}$ likely exists between 11 and 20 GPa.

Magnetic ordering of the Mn spins has an effect on the pathway of high pressure transitions. At 20 GPa, AFM type 3 ordering couples the $d$ electrons on neighboring Mn atoms with opposite spins leading to metallisation as hinted by the XANES and previous simulations. The other orderings prefer to transition into a low-spin state persistent up to at least $20 \mathrm{GPa}$. However, in each $\mathrm{Pa} \overline{3}$ structure at $11 \mathrm{GPa}$ the Mn-Mn distance is still >4 $\AA$ which is far enough to lend credence to the 2 step pathway to metallisation. Inspection of the electron localization function ${ }^{31}$ for each magnetic ordering of $\mathrm{Pa} \overline{3}$ and $P 2_{1} / c \mathrm{MnS}_{2}$ at 11 and $20 \mathrm{GPa}$ shows no build up of charge density between the line of centers of the nearby Mn atoms. This indicates that no metal-metal covalent bonds form and the $d^{5}$ electrons remain localized on the $\mathrm{Mn}$ in the low-spin states. Additionally, simulations at $50 \mathrm{GPa}$ still show low-spin $\mathrm{S}=1 / 2 \mathrm{FM}$ ordering in $\mathrm{Pa} \overline{3} \mathrm{MnS}_{2}$ is preferential to a closed-shell, metallic state by $0.65 \mathrm{eV}$ per $\mathrm{MnS}_{2}$ formula unit (H/f.u).

These results show that by $11 \mathrm{GPa}, P a \overline{3} \mathrm{MnS}_{2}$ is on the precipice of an electronic transition, although the low-spin $\mathrm{S}_{M n}=1 / 2$ state is $0.5 \mathrm{eV} \mathrm{H} /$ f.u. uphill in energy. The closed-shell, diamagnetic state appears weakly metallic and is even higher with a $\Delta \mathrm{H} / \mathrm{f} . \mathrm{u}$. of $+1.33 \mathrm{eV}$. Interestingly, the low-spin state is accompanied by a $21.5 \%$ volume collapse, $23.1 \%$ for the diamagnetic state. There are striking similarities between the low-spin $\mathrm{S}_{M n}=1 / 2$ states of $P a \overline{3}$ and $P 2_{1} / c$ at $11 \mathrm{GPa}$ : they have nearly identical volumes (for FM - 39.10 vs. $39.18 \AA^{3} / \mathrm{f} . \mathrm{u}$. respectively), they are nearly degenerate (-15.37 vs. $-15.40 \mathrm{H} /$ f.u. respectively), and they have very similar partial densities of state. Based on this evidence, it is no coincidence that the electronic transition is poised to occur at the same pressure as the transition from $P a \overline{3}$ to $P 2_{1} / c$. The electronic transition into the low-spin state triggers the large volume collapse and subsequent structural rearrangement into the more enthalpically favorable $P 2_{1} / c$ phase.

\section{CONCLUSIONS}

In summary, we have revisited the pressure induced phase transition of the pyrite mineral $\mathrm{MnS}_{2}$ using spectroscopic techniques. Our XANES measurements confirm that there is no change in oxidation state upon entering the pressure induced disordered phase at ambient temperature. Furthermore, comparison of the spectra of this phase with a crystalline arsenopyrite sample shows that the electronic structure is near identical. This shows that kinetic hindrance of a phase transition with a large volume collapse, not decomposition, is the driving force for the disordering. Finally, we note that significant electronic changes in the electronic structure of the pyrite phase are seen on compression. These include a reduction in the edge-intensity seen by XANES, a converging of the split pre-peak feature, and saturation of phonons involving the disulphide groups. While more detailed x-ray spectroscopy measurements are clearly 
justified, we end by noting that the spin -state transition in $\mathrm{MnS}_{2}$ clearly involves more variables that the octahedral crystal field. We hypothesis that band broadening may play a role, and will return to this point in a future publication.

We thank Michael Hanfland for measuring the X-ray diffraction pattern on the ID09A beam line. The ESRF is acknowledged for access to instrumentation, and M.V. Kimber and I. Zizak are thanked for their help in collecting the reference Mn XANES spectra at the BESSY-II synchrotron. Lawrence Livermore National Laboratory is operated by Lawrence Livermore National Security, LLC, for the U.S. Department of Energy, National Nuclear Security Administration under Contract DE-AC52-07NA27344. This research was sponsored in part by the National Nuclear Security Administration under the Stewardship Science Academic Alliances program through DOE Co-operative Agreement DE-NA0001982. Oak Ridge National Laboratory is managed by UT-Batelle, LLC, for the DOE under contract DE-AC05-1008 00OR22725.

* Email of corresponding author:keith.lawler@unlv.edu

$\dagger$ Email of corresponding author:salamat@physics.unlv.edu

\# Email of corresponding author:simon.kimber@u-bourgogne.fr

1 J.P. Rueff, C.-C. Kao, V.V. Struzhkin, J. Badro, J. Shu, R.J. Hemley, H.K. Mao, Phys. Rev. Lett. 82, 3284 (1999)

2 P.W. Bridgman, Proc. Am. Acad. Arts Sci. 62, 207 (1927)

${ }^{3}$ C.S. Yoo, B. Maddox, J-H.P. Klepeis, V. Iota, W. Evans, A. McMahan, M.Y. Hu, P. Chow, M. Somayazulu, D. Häusermann, R.T. Scalettar, W.E. Pickett, Phys. Rev. Lett. 94, 115502 (1995)

4 J. Kuneš, A.V. Lukoyanov, V.I.. Anisimov, R.T. Scalettar, W.E. Pickett, Nat. Mater. 7, 198 (2008)

5 W. Xiao, D. Tan, X. Xiong, J. Liu, J. Xu, Proc. Am. Acad. Arts Sci. 107, 14026 (2010)

${ }^{6}$ C. Huhnt, G. Michels, M. Roepke, W. Schlabitz, A. Wurth, Physica B 240, 26 (1997)

7 A.R. Oganov, C.W. Glass, The J. Chem. Phys. 124, 244704 (2006)

8 A. Salamat, R. Fischer, R. Briggs, M.I McMahon, S. Streltsov, D.N. Petitgirard, Coord. Chem. Rev. 277-278, 15 (2014)

9 T. Chattopadhyay, H.G. von Schnering, J. Phys. Chem. Sol. 46, 113 (1985)

10 T. Chattopadhyay, H.G. von Schnering, W.A. Grosshans, Phys. B+C 139-140, 305 (1986)

11 S.A.J. Kimber, A. Salamat, S.R. Evans, H.O. Jeschke, K. Muthukumar, M. Tomić, F. Salavat-Pujol, R. Valentí, M.V. Kaisheva, I. Zizak, T. Chatterji, Proc. Am. Acad. Arts Sci. 111, 5106 (2014)

12 S.D. Wijeyesekera, R. Hoffmann, Inorg. Chem. 22, 3287 (1983)

13 Y. Wang, L. Bai, T. Wen, L. Yang, H. Gou, Y. Xiao, P. Chow, M. Pravica, W. Yang, Y. Zhao, Angew. Chem. 55, 10350 (2016)

14 Y. Wang, Z. Zhou, T. Wen, Y. Zhou, N. Li, F. Han, Y. Xiao, P. Chow, J. Sun, M. Pravica, A.L. Corenelius, W. Yang, Y. Zhao, J. Amer. Chem. Soc. 138, 15751 (2016)

15 S. Furuseth, A. Kjekshus, Acta Chem. Scan. 19, 1405 (1965)

16 S.A.J. Kimber, T. Chatterji, J. Phys. Cond. Matt. 27, 22603 (2015)

17 V.A. Sidorov, J. Guo, L. Sun, V.V. Brazhkin, JETP Lett. 107, $311(2018)$

18 A.K. Arora, Sol. State Comm. 115, 665 (2000)

19 A. Dewaele, P. Loubeyre, M. Mezouar, Phys. Rev. B 70, 094112 (2004)

20 . Pascarelli, O. Mathon, T. Mairs, I. Kantor, G. Agostini, C. Strohm, S. Pasternak, F. Perrin, G. Berruyer, P. Chappelet, C. Clavel, M.C. Dominguez, J. Synch. Rad. 23, 353 (2016)

${ }^{21}$ I. Kantor, C. Marini, O. Mathon, . Pascarelli, Rev. Sci. Instrum. 89, 013111 (2018)

22 N. Ishimatsu, K. Matsumoto, H. Maruyama, N. Kawamura, M. Mizumaki, H. Sumiya, T. Irifume, J. Synch. Rad. 19, 768 (2012)

23 J.J. Rehr J.J. Kas F.D. Vila M.P. Prange K. Jorissen Phys. Chem. Chem. Phys. 12, 5503 (2010).

24 A. Larson and R. V. Dreele, General Structure Analysis System (GSAS), Los Alamos National Laboratory Report LAUR 86-748 (1994),

25 B.H. Toby J. Appl. Cryst. 34, 210-213 (2001).

26 P. Hohenberg, W. Kohn, Phys. Rev. 136, 864 (1964)

27 W. Kohn, L. Sham, Phys. Rev. A 140, 1133 (1965)

28 G.I. Csonka, J.P. Perdew, A. Ruzsinszky, P.H.T. Philipsen, S. Leègue, J. Paier, O.A. Vydrov, J.G. Ángyán, Phys. Rev. B 79, 155107 (2009)

29 S.L. Dudarev, G.A. Botton, S.Y. Savrasov, C.J. Humphreys, A.P. Sutton, Phys. Rev. B 57, 1505 (1998)

30 L. Wang, T. Maxisch, G. Ceder, Phys. Rev. B 73, 195107 (2006)

31 A.D. Becke, K.E. Edgecombe, J. Chem. Phys. 92, 5397 (1990)

32 H.J. Monkhorst, J.D. Pack, Phys. Rev. B 13, 5188 (1976)

33 P.E. Blöchl, Phys. Rev. B 50, 17953 (1994)

34 A. Togo, I. Tanaka, Scr. Mater. 108, 1 (2015)

35 J.F.W. Mosselmans R.A.D. Pattrick G. van der Laan J.M. Charnock D.J. Vaughan C.M.B. Henderson C.D. Garner Phys. 
Chem. Mater. 22, 311 (1995)

${ }^{36}$ M. Womes R.C. Karnatak J.M. Esteva I. Lefebvre G. Allan J. Olivier-Fourcade J.C. Jumas J. Phys. Chem. Sol. 58, 345 (1997).

37 D. Hobbs, J. Hafner, J. Phys. Cond. Matt. 11, 8197 (1999)

38 A. Rohrbach, J. Hafner, G. Kresse, J. Phys. Cond. Matt. 15, 979 (2003)

${ }^{39}$ K. Persson, G. Ceder, D. Morgan, Phys. Rev. B 73, 115201 (2006)

40 P. Vulliet, J.P. Sanchez, D. Braithwaite, M. Amanowicz, B. Malaman, Phys. Rev. B 63, 184403 (2001)

41 T. Chatterji, A.M. dos Santos, J.J. Molaison, T.C. Hansen, S. Klotz, M. Tucker, K. Samanta, T. Saha-Dasgupta, Phys. Rev. B 91, $104412(2015)$

42 H. Yamamoto, X-ray Spec. 37, $572(2008)$

43 F. Farges, Phys. Rev. B 71, 155109 (2005)

44 A. Sanson, I. Kantor, V. Cerantola, T. Irifune, A. Carnera, S. Pascarelli, Phys. Rev. B 94, $014112(2016$

45 S.S. Lobanov, H. Hsu, J-F. Lin, T. Yoshino, A. Goncharov, J. Geophys. Res. Solid Earth 122, 3565 (2017

46 S. Streltsov, A.O. Shorikov, S.L. Skornyakov, A.I. Poteryaev, D.I. Khomskii, Sci. Report 7, 13005 (2017)

47 L.A.J. Garvie, A.J. Craven, Phys. Chem. Minerals 21, 191 (1994)

48 H. Vogt, T. Chattopadhyay, H.J. Stoltz, J. Phys. Chem. Sol. 44, 869 (1983)

49 A.S. Davydof, J. Exptl. Theoret. Phys. (U.S.S.R.). 18, 210 (1948)

50 N. Lazarević, T. Radonjić, R. Hu, R. Tanasković, C. Petrovic, R. Popović, J. Phys. Cond. Matt. 24, 135402 (2012)

51 A.K. Kleppe, A.P. Jephcoat, Miner. Mag. 68, 433 (2004)

52 S. Kuze, N. Ishizawa, Bull. Soc. Disc. Var. X $\alpha$ 13, 46 (2000)

53 N. Ishizawa, Private communication, 2012.

54 Now closed, and operations moved to ID15-B 